\title{
Telemetric acquisition of vitality parameters and classification of cognitive condition via machine learning
}

\author{
Dipl.-Phys. Martin Bussas \\ TROUT GmbH, Parkstr. 28, D-34119 Kassel \\ m.bussas@trout-gmbh.de
}

\begin{abstract}
:
The ability to acquire vital parameters and classify cognitive conditions opens doors to new technologies in diverse areas such as medical technology, automation, aerospace, fitness/wellness and security. TROUT gained considerable expertise in biometric data processing during automotive and medical technology developments which focused on machine learning and Al (Artificial Intelligence). With variations in the heartbeat, the organism can respond optimally to changing endogenous and exogenous influences and thus adapt to the current needs of the blood supply. Heart rate variability (HRV) provides not only information on the degree of stress on the cardiovascular system, but also on the quality of cardiovascular regulation and has also become established in other areas in recent years, due to ever smaller measuring instruments and lower costs, as well as applications in clinical research. [1]
\end{abstract}

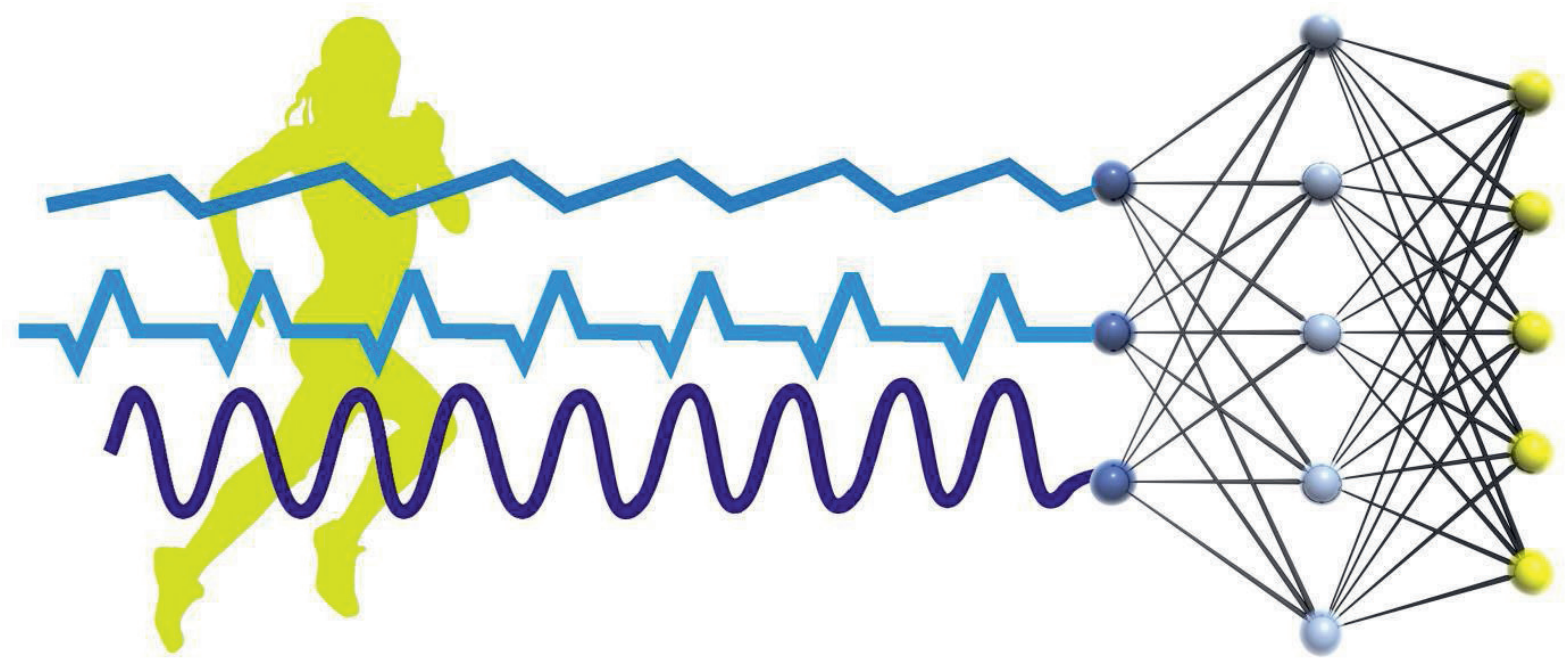

Fig. 1. Telemetric Acquisition of vitality parameters and classification

If we add information about the activities of an individual in correlation with their vital data, and process both data through a machine learning system, we are now able to achieve very good results concerning the individual's cognitive state such as stress level and fatigue. The system is adjusted by a feed-back loop mapping the individual's self-estimation of their cognitive state.

Key words: Artificial Intelligence, Machine Learning, Cognitive State, Stress Level.

\section{Introduction}

TROUT's R\&D project VitaB focuses on the telemetric acquisition of vital data. VitaB is the German abbreviation of 'Determination of Vitality Parameters - Vitalitätsdatenbestimmung'. Telemetric sensors are e.g. video cameras, IR sensors and high frequency probe heads for heart rate and respiration rate.
VitaB was initiated by TROUT, and is funded in the framework of Hessen ModellProjekte (HA project no. 545/17-27), by LOEWE - LandesOffensive zur Entwicklung Wissenschaftlichökonomischer Exzellenz, Förderlinie 3: KMUVerbundvorhaben (State Offensive for the Development of Scientific and Economic Excellence). 
One major focus is on investigating the relation between internal tension and external workload. Another objective of the R\&D project is to establish a driver's state management system by classifying the driver's emotional and mental state and determining needs-based influencing measures targeting an optimal state. Machine learning algorithms detect relevant patterns in physiological and visual data. These patterns are used to classify the driver's condition at different levels of cognitive workload, arousal, valence and vigilance.

To support the development of a multilevel algorithm, an analysis application has been established which visualizes data and detects and evaluates apparent conspicuities. Several filters and evaluation functions are used to normalize and smooth the data for plausibility checks. The initial assumption is verified and optimized with technical and scientific support from the medical field, including a cardiovascular center amongst others. The resulting multilevel classification showed the desired high quality results, which were then further evaluated.

The objective of VitaB is to develop a crosssectional, parameter-driven system that evaluates user vitality in order to classify the corresponding cognitive condition. Manifold products can be derived from the functional prototype. Using a closed-loop function, the estimated user state is continuously inspected and if necessary, positively influenced by downstream of adaptive systems. Depending on the estimate, an adaptive system can act in different ways in order to positively support the user.

\section{Simulation Environment}

3D goggles - not visible in the picture below are used for perfect immersion into the driving environment.

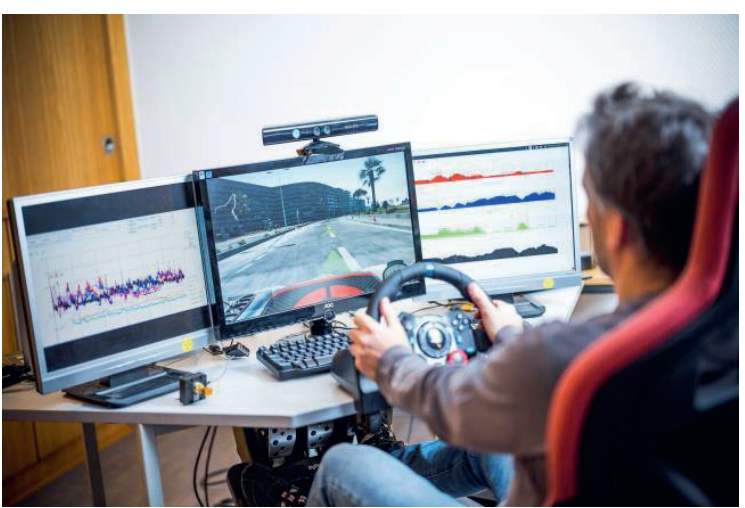

Fig. 2. Simulator, photo HA - Jan Michael Hosan

Furthermore, we use a steering wheel equipped with motion sensors which detect acceleration and velocity of the steering motions of the driver. Gear stick and pedals complete the driving environment.

\section{ECG Sensor}

The electrocardiogram (ECG) recording above illustrates beat-to-beat variability in $\mathrm{R}-\mathrm{R}$ interval (top) and heart rate (bottom). Heart rate variability (HRV) is the physiological phenomenon of variation in the time interval between heartbeats. It is measured by the variation in the beat-to-beat interval.

Other terms used include: "cycle length variability", "RR variability" (where $R$ is a point corresponding to the peak of the QRS complex of the ECG wave, and RR is the interval between successive Rs), and "heart period variability". The analysis of heart rate variability (HRV) has evolved in recent years to an established non-invasive detection of the level of exercise of the cardiovascular system. Thus, the HRV describes the interaction between the sympathetic and parasympathetic nervous system, which controls the activity of the heart, and allows the investigator to mathematically attribute the influence of the autonomic nervous system to the regulation of the cardiovascular system. This review is concerned with the analysis methods of HR-related, frequencyrelated and non-linear methods. Notes on the selection of the meaningful detection period are discussed and the visual values are presented for selected HRV parameters. [2] [3] [7].

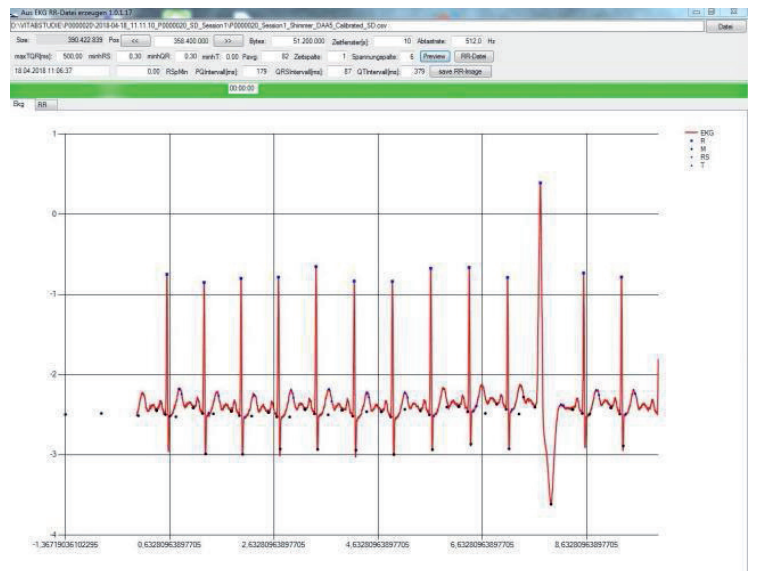

Fig. 3. ECG software to extract the R-R intervals

The ECG software, which extracts the R-R intervals from the signal, has been developed by TROUT. As the diagram above shows, the software has to deal with irregularities in the heart beat which occur once in a while.

\section{Telemetric ECG sensor}

In the previous study, we were able to evaluate a telemetric radar based ECG sensor from HF Systems Engineering $\mathrm{GmbH}$ \& Co. KG with great success. 
The basic idea of the radar based accurate heartbeat estimation method consists of timedomain signal processing applied on the electromagnetic waves reflected from the chest of the subject. A wideband signal in the middle region of the mm-wave band is used as the sounding signal, which is coherently recorded at the transceiver. Hence, the signal does not propagate through the tissue. Instead it scans precisely the skin movement due to respiration and micro skin vibration due to the heartbeat. Thus, a superposition of the strong chest signal and the weak heart signal resembles the raw data.

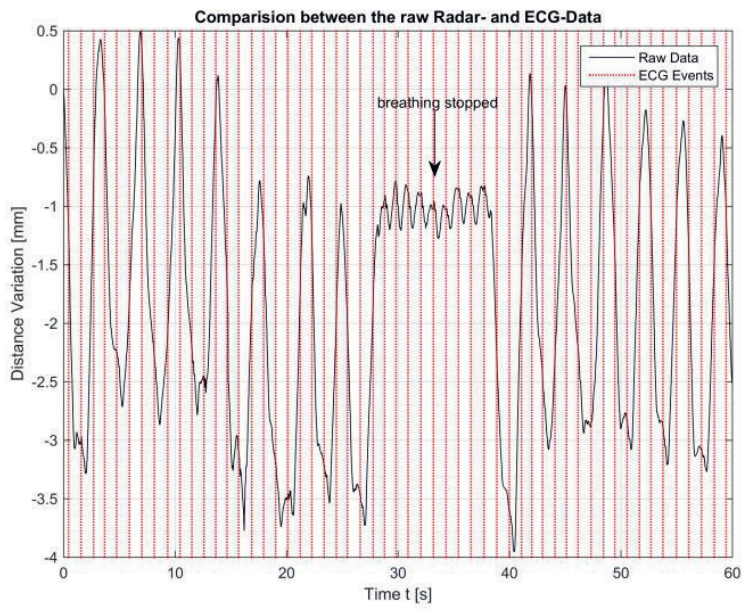

Fig. 4. Radar pulse versus ECG pulse

Super-resolution methods for highly accurate distance estimations are well known from specialist journals and documentation and are applied here to extract distance information of the range cell in the air-skin boundary. Subsequently, the distance vector is processed with spectral estimation methods and time domain parameter estimation methods to finally separate the breathing contamination from the heart beat content.

The radar signal reaches sufficient precession to be used for stress level evaluation below.

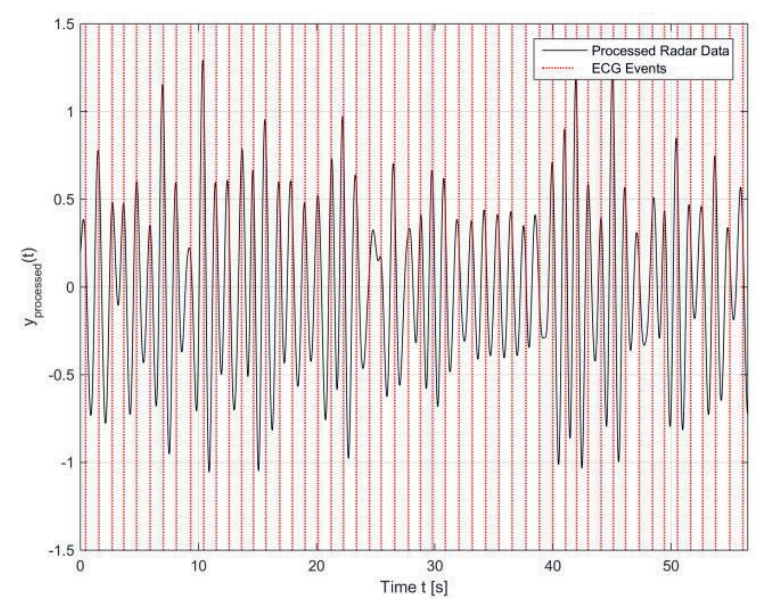

Fig. 5. Processed radar data

\section{Acceleration Sensor in the Steering Wheel}

Besides breath and heartbeat signals, we evaluate speed and acceleration via the steering wheel. This information is fed into the machine learning algorithms after processing the physiological data through a physiological model.

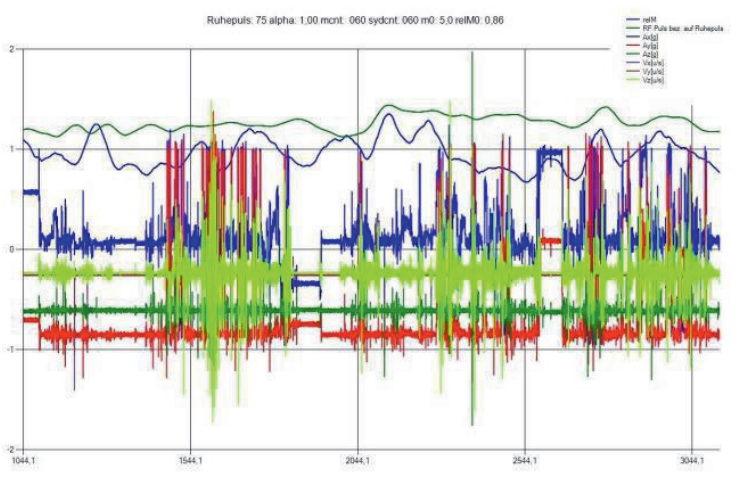

Fig. 6. Paired pulse ratio, steering wheel velocity and steering wheel acceleration versus time [s]

\section{Physiological Model}

The following features are extracted from available ECG data:

1. Actual pulse related to the resting pulse of the subject.

2. Average change in heart rate in percent per second averaged over 60 seconds, based on the average value determined for the subject.

3. Ratio of the velocity of pulse acceleration to the velocity of pulse deceleration averaged over 60 seconds, which is an indicator of the relationship between sympathetic and parasympathetic activity. This value is then calibrated according to the subject.

4. Ratio LF / HF from the Fourier Analysis determined ratio of the amplitude sums for LF and HF.

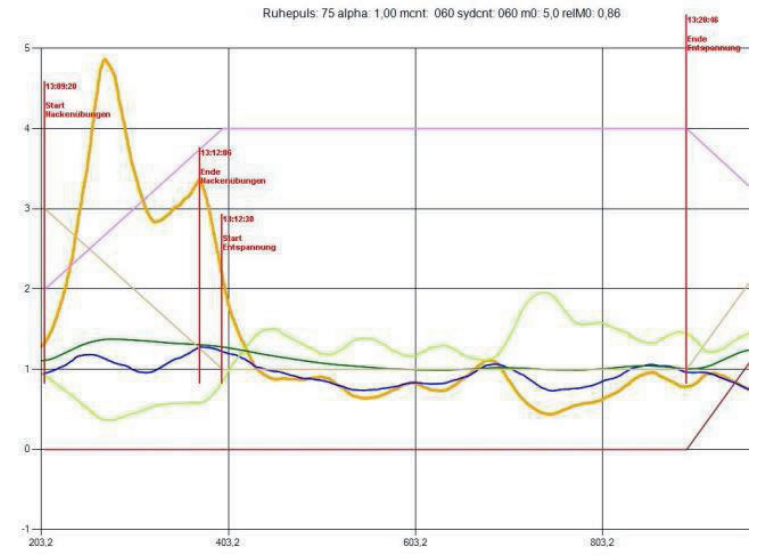

Fig. 7. Relaxation exercise 
Legend for fig. 7 to 10: Heart rate based on the resting heart rate (green), ratio of pulse increases to pulse decreases (blue), HRV Heart Rate Variability (light green), Event Marker (red - in German language), Calculated Stress Level (yellow) in a relative scale from 0 (no stress) to 7 (maximum stress) on the ordinate, the abscissa shows the time in seconds.

The drive simulation starts with a relaxation exercise in order to allow the subject to lower his pulse rate to the rest pulse rate.

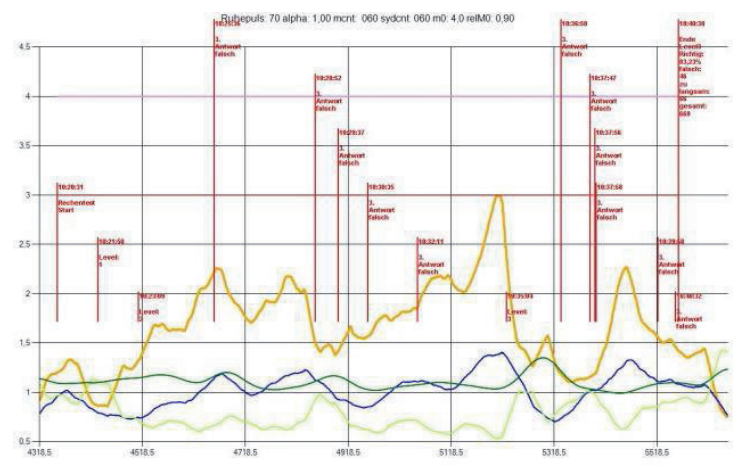

Fig. 8. Math Test

To increase the stress level, we start a math test after a couple of minutes until the subject has become accustomed to the operation of the simulation stand. The calculation starts with very easy tasks. With each level, the tasks become more difficult and the required response times are reduced. Each achieved level is logged along with frequent incorrect answers and a buzzer signal is activated.

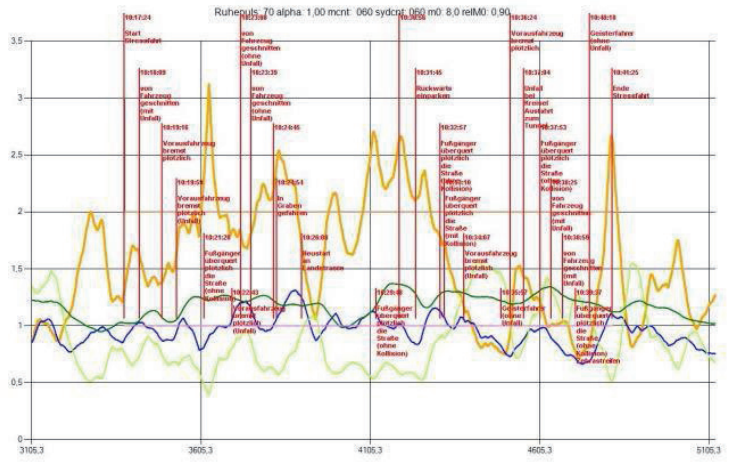

Fig. 9. Rush Hour

After the math test, the subject drives along a quiet mountain road before entering the rush hour traffic of a busy city. As the event markers above indicate, there are tasks waiting such as cars on the wrong side of the road or children running behind parked cars.

It is obvious that the calculated stress level yellow line - meets the expectations such as high stress level shortly after 'near accident' situations and low stress level after the mountain ride tour and when the rush hour events terminate.

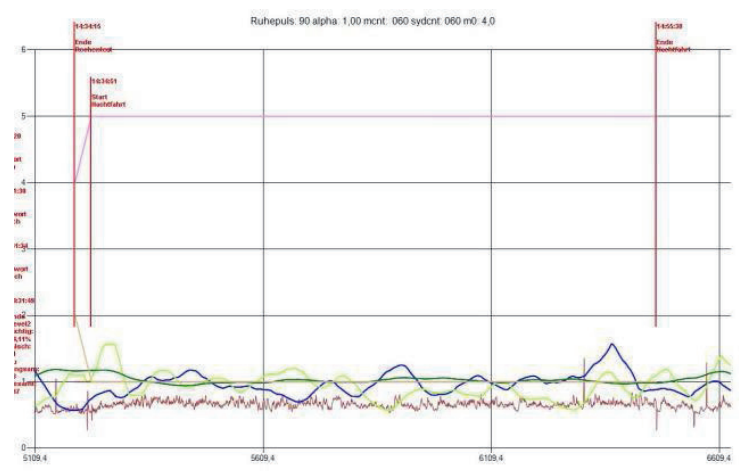

Fig. 10. Night Ride

Towards the end of the drive study, after almost two hours of strain, there are twenty more minutes to go on an empty motorway in the twilight which trigger tiredness in the end.

\section{Machine Learning}

The sensor signals and outcome of the physiological model are classified by means of various methods from the field of machine learning. The accuracy is enhanced by applying ensemble techniques, i.e. a dynamic combination of the following techniques. [4]

\section{Artificial Neural Networks}

Artificial Neural Networks (ANNs) are artificial neuron networks. Artificial neural networks, like artificial neurons, have a biological role model. They are juxtaposed with the natural neural networks that form nerve cell networks in the brain and spinal cord. However, ANNs are more about abstraction (modeling) of information processing.

\section{Convolutional Neural Networks}

Convolutional Neural Networks (CNNs) employ a variation of multilayer neurons designed to require minimal preprocessing. They are also known as space-invariant artificial neural networks based on their shared weights architecture and translation invariance characteristics. Here CNNS are used for feature classification after preprocessing the raw vital data using a physiological model. [5]

\section{Random Forest}

The method of Random Forest is a classification procedure consisting of several uncorrelated decision trees. All the decision trees have developed under a certain type of randomization during the learning process. Decision trees are ordered, directed trees that serve to represent decision rules. The graphical representation as a tree diagram illustrates successive decisions hierarchically. 


\section{Support Vector Machines}

In machine learning, Support Vector Machines (SVMs) are supervised learning models with associated learning algorithms that analyze data for classification and regression analysis. In a series of training examples, each labeled as belonging to one of the two categories, an SVM training algorithm creates a model that assigns new examples to one or the other category, thereby creating a non-probabilistic binary linear classifier.

A Support Vector Machine serves as a classifier and regressor. It subdivides a set of objects into classes so that the broadest possible range remains free of objects around the class boundaries, the so-called large margin classifier. This is a mathematical method of pattern recognition from the field of machine learning. The basis for building a Support Vector Machine is a set of training objects, each of which knows which class to belong to. Each object is represented by a vector in a vector space. The task of the Support Vector Machine is to fit a hyperplane into this space that acts as the interface and divides the training objects into two classes. The distance of those vectors closest to the hyperplane is thereby maximized. This wide, empty border will ensure that even objects that do not correspond exactly to the training objects are classified as reliably as possible. [6]

\section{Derived Values}

After feature extraction, an automatic feature selection was used. Filter or wrapper approaches can both be used for feature selection. Filter approaches evaluate features based solely on characteristics that can be calculated from the data. Wrapper approaches use a classification algorithm (often the target algorithm that is also used in the final application) and evaluate features according to their contribution to the performance. The former approaches have the advantage that they are easy to calculate, while the latter have the advantage of providing very problem specific results.

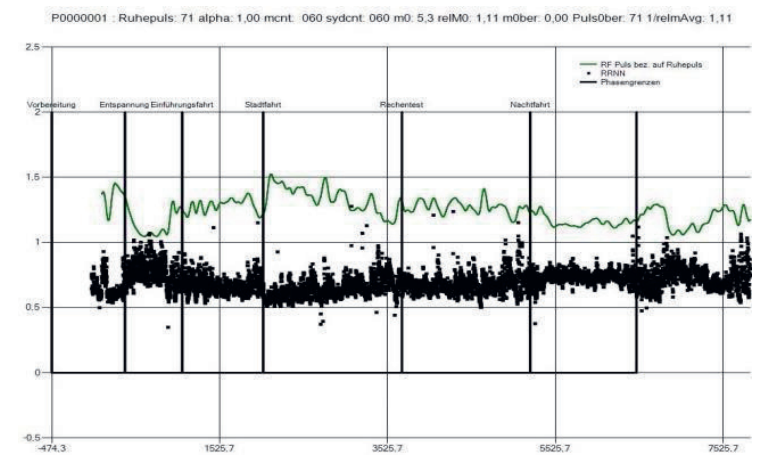

Fig. 11. RF and normalized R-R intervals RRNN
Fig. 11 shows the pulse related to the resting pulse of the subject RF and the normalized R-R intervals. At rest, RF is lowest, it is heavily elevated during physical activity and slightly increased in mental activity.

Fig. 12 shows the average change in the heart rate in percent per second averaged over 60 seconds based on the average value determined for the subject $(\mathrm{m})$. It is low during activity. The value is related to the average pulse value of the subject.

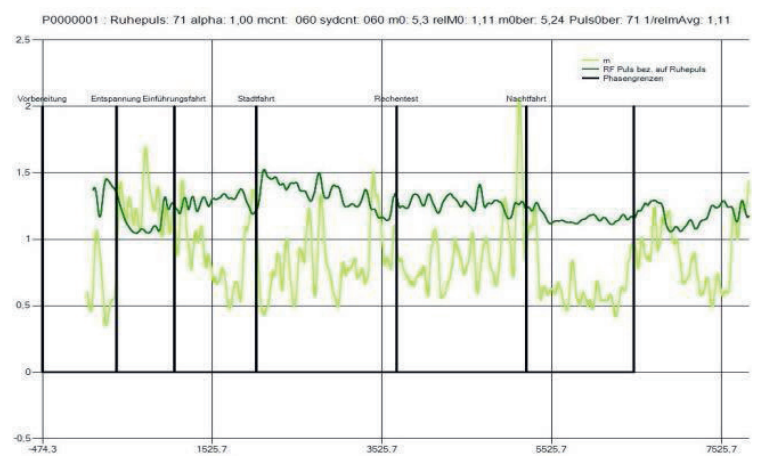

Fig. 12. $m$

The ratio of the rates of pulse acceleration to the rates of pulse deceleration averaged over 60 seconds (RelM). Measurement of the relationship between sympathetic and parasympathetic activity. The value is set by a factor that averages a value of 1 .

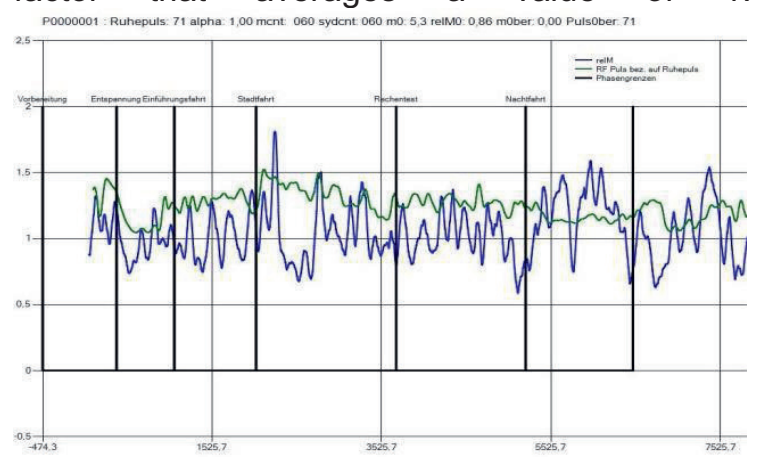

Fig. 13. RelM

Strong fluctuations indicate increased activity and stress. When tired, it is below 1 and does not fluctuate much.

Stress is likely with pulse higher, RelM higher and $\mathrm{m}$ lower. Fatigue can be expected with pulse lower and relM not fluctuating so very much.

\section{Results}

Fig. 14 on the next page shows an example of the trend of the vital parameters, derived parameters and calculated stress level and fatigue for a test period of more than two hours.

The concordance with the subjects' selfassessment is greater than $80 \%$. 
Ruhepuls: 75 alpha: 1,00 mcnt: 060 sydcnt: 060 m0: 10,0 relM0: 0,86

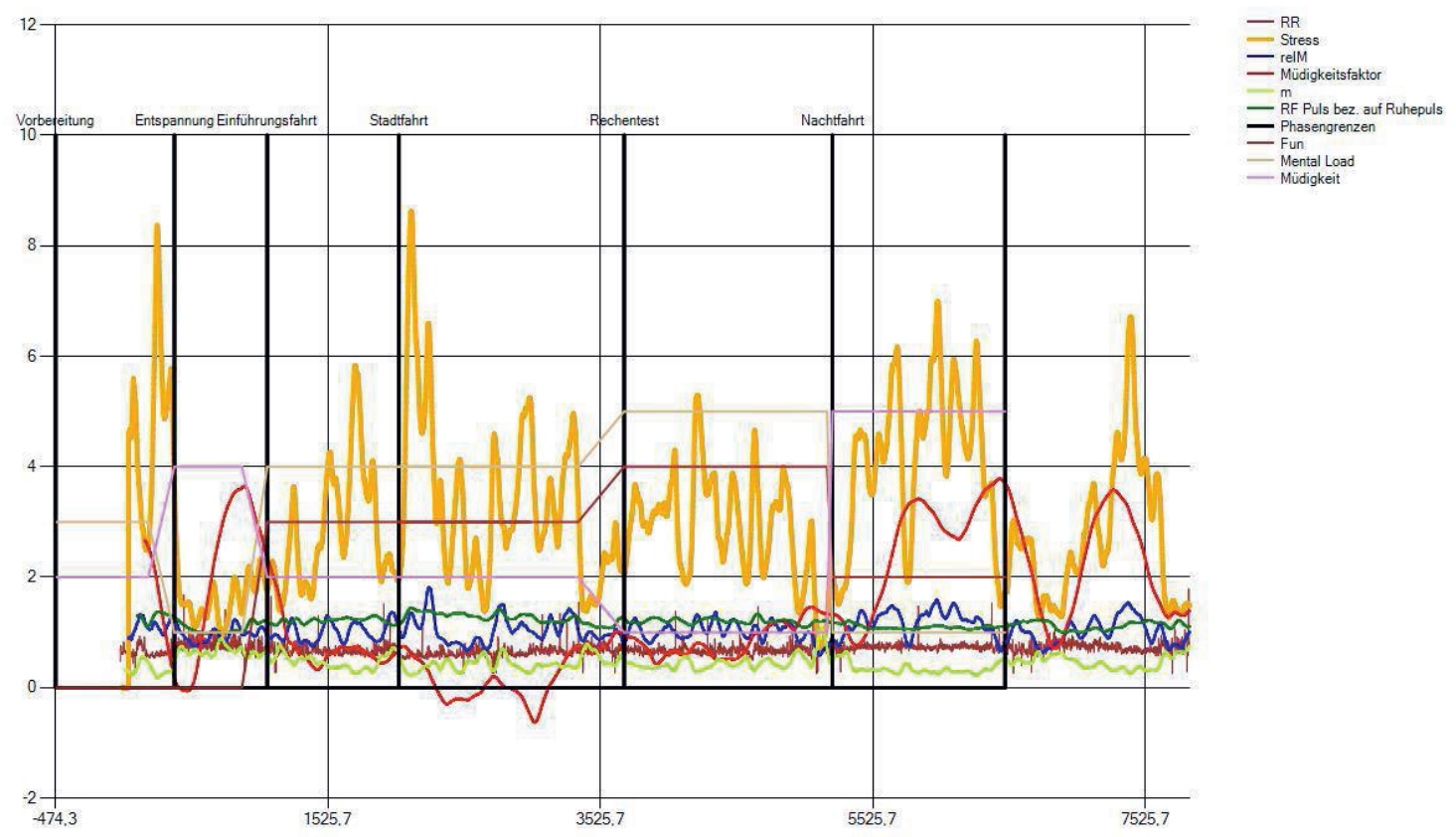

Fig.14. Stresslevel (yellow) and Fatigue (pink) versus drive time and events

\section{Conclusions}

Adaptations to new requirements, sensors and interfaces are easily implemented and the embedded environment features low current consumption and hardware costs, thus meeting applicable standards (e.g. electromagnetic compatibility, software and hardware development, safety and security). Furthermore, a real time learning feature has been developed and implemented.

Products or derivatives will be based on the functional prototype. In this context, the following areas pose further fields of application: automation, aerospace (e.g. 'intelligent' pilot and / or passenger seats or seats for tower staff), medicine, wellness / fitness, as well as security (person recognition, state evaluation).

Using a closed-loop function, the estimated user state is continuously inspected and, if necessary, positively influenced by downstream of adaptive systems. Depending on the current estimate, an adaptive system can act in different ways in order to assist the user to regain a good state. For instance, one such action is to adapt the interior air conditioning of a vehicle.

\section{References}

[1] Prof. Dr. med. Kuno Hottenrott Herzfrequenzvariabilität im Sport; Hamburg; Czwalina, pp. 9-26

[2] S. Sammito / I. Böckelmann: Analyse der Herzfrequenzvariabilität Herz 2015-40576-S84-DOI 10.1007/s00059-0144145-7; Urban \& Vogel 2014

[3] Task Force of The European Society of Cardiology and The North American Society of Pacing and Electrophysiology (Membership of the Task Force listed in the Appendix) European Heart Journal (1996) pp. 17, 354- 381

[4] Christopher Bishop, Pattern Recognition and Machine Learning, Springer, 2006

[5] TensorFlow: OpenSource Machine learning framework; https://tensorflow.org

[6] OpenCV: OpenSource Computional Vision Library; https://opencv.org

[7] PhysioNet: the research resource for complex physiologic signals; https://physionet.org 